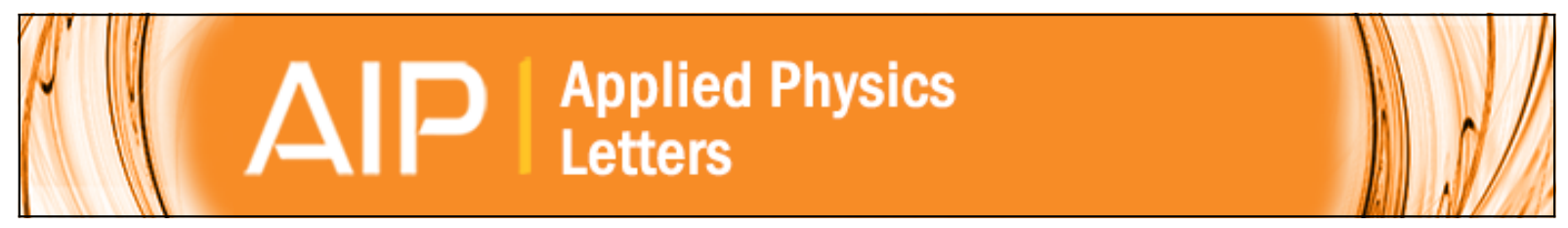

\title{
Ambipolar organic field-effect transistors fabricated using a composite of semiconducting polymer and soluble fullerene
}

Shinuk Cho, Jonathan Yuen, Jin Young Kim, Kwanghee Lee, and Alan J. Heeger

Citation: Applied Physics Letters 89, 153505 (2006); doi: 10.1063/1.2361269

View online: http://dx.doi.org/10.1063/1.2361269

View Table of Contents: http://scitation.aip.org/content/aip/journal/apl/89/15?ver=pdfcov

Published by the AIP Publishing

\section{Articles you may be interested in}

Ambipolar pentacene/ C 60 -based field-effect transistors with high hole and electron mobilities in ambient atmosphere

Appl. Phys. Lett. 94, 023305 (2009); 10.1063/1.3072608

Ambipolar charge carrier transport in mixed organic layers of phthalocyanine and fullerene

J. Appl. Phys. 101, 063709 (2007); 10.1063/1.2436836

Organic heterostructure field-effect transistors using $C 60$ and amorphous spirolinked compound Appl. Phys. Lett. 89, 253516 (2006); 10.1063/1.2423248

High performance $\mathrm{n}$-channel organic field-effect transistors and ring oscillators based on $\mathrm{C} 60$ fullerene films Appl. Phys. Lett. 89, 213504 (2006); 10.1063/1.2387892

Fabrication of ambipolar field-effect transistor device with heterostructure of $\mathrm{C} 60$ and pentacene Appl. Phys. Lett. 85, 4765 (2004); 10.1063/1.1818336

A P Pplied Physics A Letters

is pleased to announce Reuben Collins as its new Editor-in-Chief

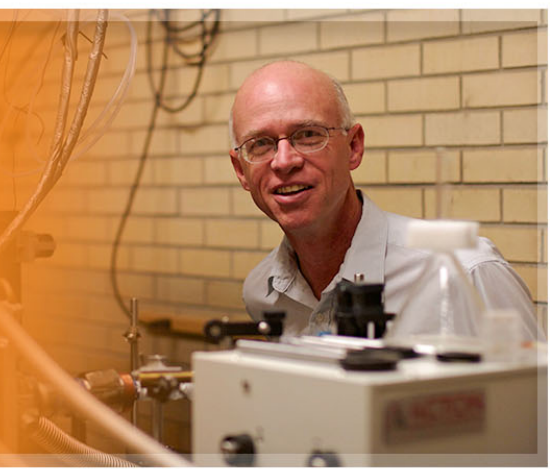




\title{
Ambipolar organic field-effect transistors fabricated using a composite of semiconducting polymer and soluble fullerene
}

\author{
Shinuk Cho, Jonathan Yuen, Jin Young Kim, ${ }^{a)}$ Kwanghee Lee,, b) and Alan J. Heeger \\ Center for Polymers and Organic Solids, University of California at Santa Barbara, \\ Santa Barbara, California 93106-5090
}

(Received 20 July 2006; accepted 31 August 2006; published online 11 October 2006)

\begin{abstract}
Organic field-effect transistors (FETs) with equivalent hole and electron mobilities have been demonstrated. The devices were fabricated using a phase separated mixture of regioregular poly(3-hexylthiophene) and [6,6]-phenyl $\mathrm{C}_{61}$-butyric acid methyl ester as the active layer and using aluminum (Al) for the source and drain electrodes. Measurements of the source-drain current versus gate voltage gave an electron mobility of $\mu_{e}=2.0 \times 10^{-3} \mathrm{~cm}^{2} / \mathrm{V} \mathrm{s}$ and hole mobility of $\mu_{h}=1.7$ $\times 10^{-3} \mathrm{~cm}^{2} / \mathrm{V}$ s. The ambipolar FET properties arise from the use of Al electrodes for the source and drain; the contacts between the $\mathrm{Al}$ electrodes and the active layer are improved by thermal annealing at elevated temperatures $\left(150{ }^{\circ} \mathrm{C}\right)$, thereby enabling balanced injection for both holes and electrons in a single device. () 2006 American Institute of Physics. [DOI: 10.1063/1.2361269]
\end{abstract}

Polymer field-effect transistors (FETs) show promise as the critical components for low-cost, flexible electronics with various applications including smart cards, electronic identification tags, and active matrix displays. ${ }^{1-4}$ These applications typically require complementary logic elements, which require both $p$-type and $n$-type transistors. In such a case, it is beneficial to utilize "ambipolar" FETs, which function both as $p$-channel and $n$-channel transistors and, therefore, reduce the complexity of device fabrication. ${ }^{2,5-7}$ There are, however, no examples in the literature of "genuine" ambipolar organic FETs which exhibit comparable $n$-type and $p$-type mobilities, because typical organic materials show either higher hole mobility or higher electron mobility. ${ }^{8-12}$

In efforts to realize ambipolar organic FETs, researchers have attempted to use different metals for the source and drain electrodes, ${ }^{13}$ to use blend materials as the active layer in the channel, ${ }^{7,14-16}$ or to use bilayers consisting of hole transporting and electron transporting materials. ${ }^{1,17,18}$ The use of blend materials (comprised of hole and electron transporting materials) appears to be most promising because of several advantages including easy fabrication of the active semiconducting layer from solution and the use of a single metal for the source and drain electrodes.

Although various blend materials have been tried for ambipolar organic FETs, ${ }^{7,14-16}$ the electron mobilities are typically lower than the hole mobilities. Moreover, devices with a high proportion of n-type material exhibit limited lifetimes because typically $n$-type organic materials are sensitive to ambient air and moisture. Another reason for the poor performance of $n$-channel devices originates from the use of gold $(\mathrm{Au})$ as the source and drain electrodes in typical organic FETs. Au is not suitable for ambipolar FETs because, in most cases, the work function of $\mathrm{Au}$ is too high to allow electron injection into the lowest unoccupied molecular orbital (LUMO) of the electron transporting material. Therefore, it is of importance to select a suitable metal that will enable both hole and electron injection holes in ambipolar organic FETs.

\footnotetext{
a) Also at Department of Physics, Pusan National University, Busan 609-735, Korea.

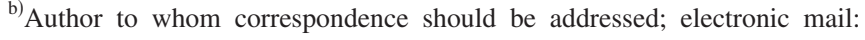
kwhlee@pusan.ac.kr
In this work, we have demonstrated excellent ambipolar performance in organic FETs fabricated with the bulk heterojunction (BHJ) material used previously in studies of solar cells fabricated from semiconducting polymers, i.e., a 1:1 mixture of regioregular poly(3-hexylthiophene) (rr-P3HT) and [6,6]-phenyl $\mathrm{C}_{61}$-butyric acid methyl ester (PCBM) as the active layer. Aluminum (Al) metal, used for the source and drain electrodes, yields good injection into both components of the phase separated BHJ composite. Postproduction heat treatment improves the contact between the $\mathrm{Al}$ electrodes and active layer; the thermal annealing also improves the morphology and crystallinity of the phase separated components (rr-P3HT and PCBM) of the BHJ material. The resulting devices exhibit an electron mobility of $\mu_{e}=2.0$ $\times 10^{-3} \mathrm{~cm}^{2} / \mathrm{V} \mathrm{s}$ and an equivalent hole mobility of $\mu_{h}=1.7$ $\times 10^{-3} \mathrm{~cm}^{2} / \mathrm{V} \mathrm{s}$.

Polymer FETs were fabricated on a heavily doped $n$-type $\mathrm{Si}$ wafer with a $200 \mathrm{~nm}$ thick thermally grown $\mathrm{SiO}_{2}$ layer. The doped $n$-type $\mathrm{Si}$ functions as a gate electrode and the oxide layer as the gate dielectric. Source and drain electrodes (Al) with $50 \mathrm{~nm}$ thickness were deposited onto the $\mathrm{SiO}_{2}$ by e-beam evaporation; the electrodes were patterned using standard photolithographic methods. All FET devices were made with bottom contact geometry. The channel length of the devices was $5 \sim 10 \mu \mathrm{m}$ and the channel width was $1 \mathrm{~mm}$. Before deposition of the P3HT-PCBM bulk heterojunction films, the $\mathrm{Al}$ electrodes were etched with standard aluminum etchant to remove the $\mathrm{Al}_{2} \mathrm{O}_{3}$ layer.

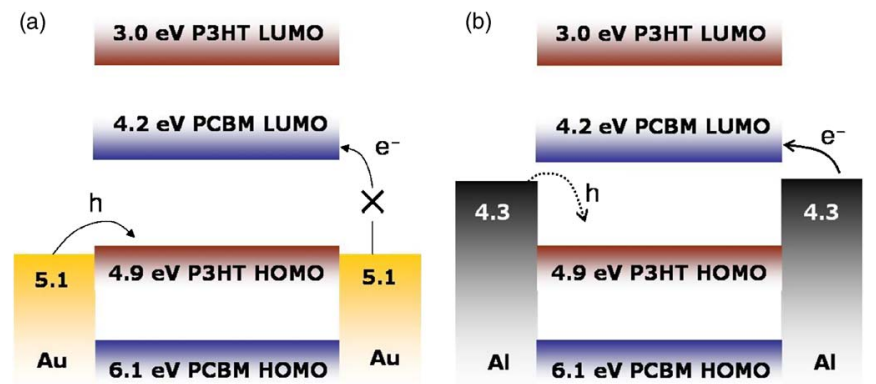

FIG. 1. (Color online) Energy level diagrams of P3HT-PCBM in contact with (a) Au or (b) $\mathrm{Al}$ electrodes under no applied bias to the transistor. 

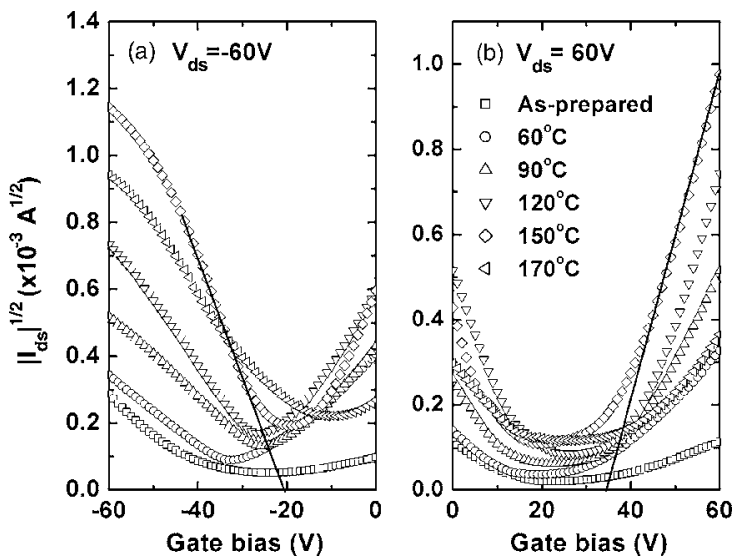

FIG. 2. Transfer characteristics of ambipolar FETs $\left(\left|I_{\mathrm{ds}}\right|^{1 / 2}\right.$ vs $\left.V_{\mathrm{gg}}\right)$ using a 1:1 ratio of $\mathrm{P} 3 \mathrm{HT}$ and $\mathrm{PCBM}$ on $\mathrm{Al}$ source-drain electrodes at various annealing temperatures: (a) $p$-type operation mode $\left(V_{\mathrm{ds}}=-60 \mathrm{~V}\right)$ and (b) $n$-type operation mode $\left(V_{\mathrm{ds}}=60 \mathrm{~V}\right)$. The solid lines drawn through the data obtained after annealing at $150{ }^{\circ} \mathrm{C}$ yield $\mu_{e}=2.0 \times 10^{-3} \mathrm{~cm}^{2} / \mathrm{V} \mathrm{s}$ and $\mu_{h}$ $=1.7 \times 10^{-3} \mathrm{~cm}^{2} / \mathrm{V} \mathrm{s}$.

Active layers were deposited by spin casting at $3000 \mathrm{rpm}$ for $60 \mathrm{~s}$ from a chlorobenzene solution containing P3HT-PCBM (1:1 ratio) with a concentration of 0.5 wt. \%. The thickness of the active layer was around $60 \mathrm{~nm}$. Thermal annealing was carried out on a calibrated and stabilized hot plate under $\mathrm{N}_{2}$ atmosphere. After annealing, the FETs were put on a metal plate at room temperature for cooling to room temperature. Electrical characterization was performed using a Keithley semiconductor parametric analyzer (Keithley 4200) under $\mathrm{N}_{2}$ atmosphere.

Figure 1 shows schematic energy level diagrams of the component materials using $\mathrm{Au}$ electrodes [Fig. 1(a)] and Al electrodes [Fig. 1(b)]. In order to achieve ambipolar FET performance using the P3HT-PCBM mixture, these diagrams demonstrate that the work function of the electrode should be selected to allow an easy hole injection into the highest occupied molecular orbital (HOMO) of P3HT and also allow an electron injection into the LUMO of PCBM. With $\mathrm{Au}$ electrodes, holes can be readily injected into the HOMO of P3HT, but electron injection would be difficult because of the large energy barrier between the Au electrode and the LUMO of PCBM.

On the other hand, for devices fabricated with $\mathrm{Al}$ electrodes, although the energy barrier between the work function of $\mathrm{Al}$ and the HOMO of P3HT is greater than that with $\mathrm{Au}$, the hole currents are sufficiently large to support ambipolar performance. Electron injection is significantly better than with $\mathrm{Au}$ electrodes because the work function of $\mathrm{Al}$ is much closer to the LUMO of PCBM [see Fig. 1(b)]. Therefore, devices using $\mathrm{Al}$ electrodes are expected to show ambipolar FET properties.

Figure 2(a) shows the transfer characteristics of the devices at various annealing temperatures $(T)$. In the case of the as-prepared device in a $p$-type operating mode [see Fig. 2(a)], the hole current gradually increases as the device is annealed from room temperature (RT) up to $150{ }^{\circ} \mathrm{C}$, then decreases for annealing temperatures $T>150{ }^{\circ} \mathrm{C}$. A similar effect of annealing is observed for the $n$-type operation [see Fig. 2(b)].

Figure 3 exhibits typical ambipolar characteristics of FETs in the plots of drain-source current $\left(I_{\mathrm{ds}}\right)$ versus drainsource voltage $\left(V_{\mathrm{ds}}\right)$ for $p$-type operation (Fig. 3(a): as prepared; Fig. 3(c): heat annealed at $150{ }^{\circ} \mathrm{C}$ ) and for $n$-type operation (Fig. 3(b): as prepared; Fig. 3(d): heat annealed at $150{ }^{\circ} \mathrm{C}$ ). For the low gate voltages, the devices show diodelike curves, which are observed frequently in typical ambipolar transistors. This feature occurs because of the presence of both charge carriers (holes and electron) in the channel of ambipolar FETs. The threshold voltages are $\left|V_{\mathrm{gs}}\right| \approx 35 \mathrm{~V}$ for $n$-channel operation and $\left|V_{\mathrm{gs}}\right| \approx 20 \mathrm{~V}$ for $p$-channel operation. The relatively high threshold voltages imply electron and hole trapping, respectively, in the PCBM and P3HT
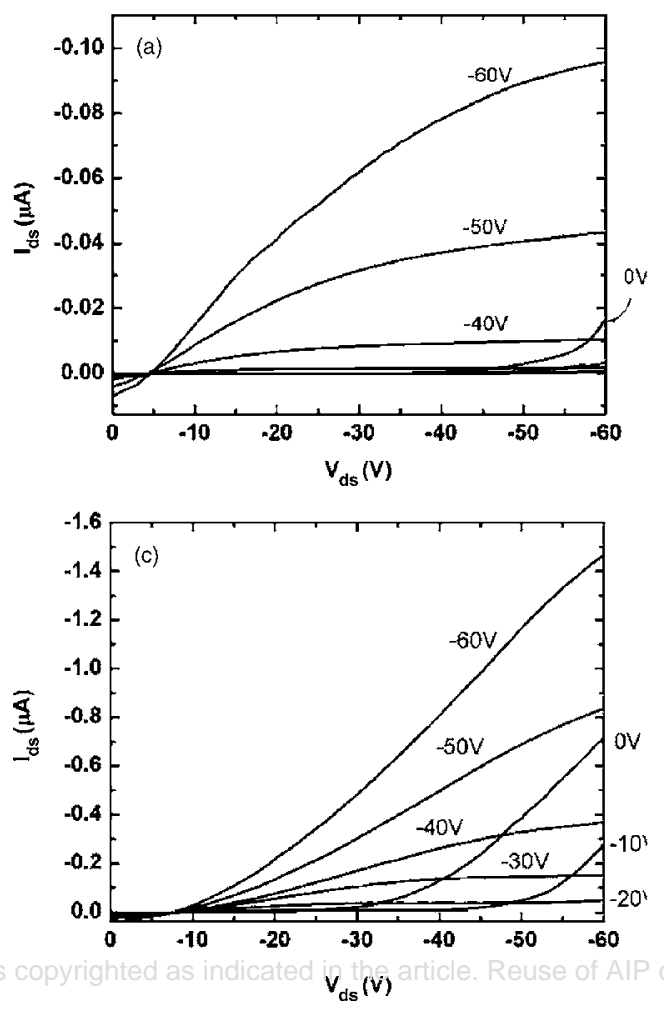

FIG. 3. Typical $I_{\mathrm{ds}}-V_{\mathrm{ds}}$ characteristics for the ambipolar FETs using a P3HT/ PCBM mixture and Al source-drain electrodes: (a) p-type operation of asprepared device, (b) n-type operation of as-prepared device, (c) p-type operation of annealed device at $150{ }^{\circ} \mathrm{C}$ for $10 \mathrm{~min}$, and (d) n-type operation of annealed device at $150{ }^{\circ} \mathrm{C}$ for $10 \mathrm{~min}$. 


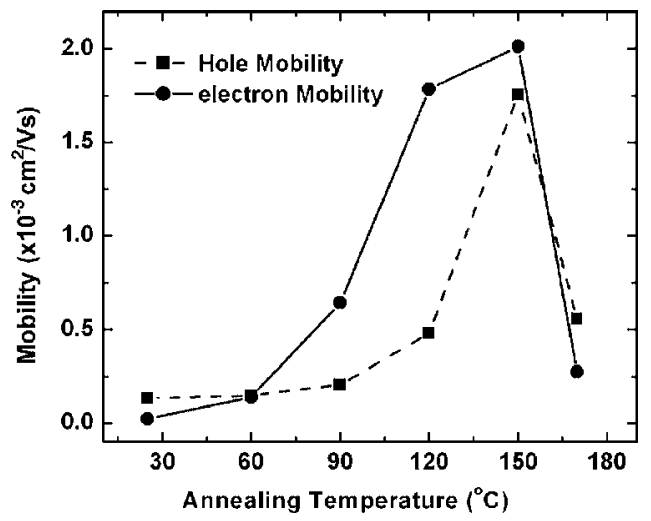

FIG. 4. Annealing temperature dependence of the electron and hole mobilities.

components (or at the interface between the two) in the phase separated network. At high gate voltages, true $p$ - or $n$-channel behavior begins to appear. The opposite currents in the output characteristics at $V_{\mathrm{ds}}=0$ seem to originate from some gate leakage current which is more prominent in the large gate bias. In contrast with the nonannealed cases, however, we notice that the output curves of the annealed samples did not exhibit the saturated feature. Although we do not completely understand this reason at this moment, we consider that it might be associated with the short-channel effect as reported by Chabinyc et al. ${ }^{19}$

Based on this observation, for the as-prepared devices, hole transport is dominant over electron transport. On the other hand, after annealing at $150{ }^{\circ} \mathrm{C}$, both hole and electron currents are enhanced. In particular, the $n$-type transport significantly improves and becomes almost comparable to the $p$-type transport. In addition, the onset voltage required for injection in the heat-annealed device at zero gate bias is lower than that of the as-prepared device. Since the injection voltage of ambipolar FETs in zero gate bias is affected by the contact resistance between the polymer and the electrode and by the energy level difference between the work function of metal and the HOMO or the LUMO of the active layer, the lower injection voltage of the heat-annealed device implies improved contact of both the phase separated components to the $\mathrm{Al}$ electrode. This phenomenon is consistent with previously published results. ${ }^{20,21}$

Figure 4 shows the mobilities $(\mu)$ as a function of the annealing temperature $(T)$. Both the hole and electron mobilities increase with annealing temperature from RT up to $150^{\circ} \mathrm{C}$. The hole mobility improves from $1.3 \times 10^{-4}$ to 1.7 $\times 10^{-3} \mathrm{~cm}^{2} / \mathrm{V} \mathrm{s}$ after annealing at $150{ }^{\circ} \mathrm{C}$; the electron mobility increases by almost two orders of magnitude from $2.2 \times 10^{-5}$ to $2.0 \times 10^{-3} \mathrm{~cm}^{2} / \mathrm{V} \mathrm{s}$. For annealing at $T$ $>150{ }^{\circ} \mathrm{C}$, the mobilities sharply decrease, consistent with data obtained from unipolar FETs using rr-P3HT. ${ }^{21}$ In these earlier studies, it was proposed that annealing at $T$ $\leqslant 150{ }^{\circ} \mathrm{C}$ improves the rr-P3HT fibrillar length and morphology constructed with the $\pi$ - $\pi$ stacked lamellar structure, thereby improving the field-effect hole mobility. ${ }^{21,22}$ For $T$ $>150{ }^{\circ} \mathrm{C}$, these fibrils decompose into small grains along the fibril axis. Therefore, the hole mobility sharply decreases at $T>150{ }^{\circ} \mathrm{C}$.

Thermal annealing also strongly influences the crystallinity of the PCBM component. Annealing at high temperature leads to demixing and more complete phase separation, resulting in the formation of nanometer-sized PCBM clusters and microsized crystals. ${ }^{22-24}$ Therefore, the rapid decrease of the electron mobility at $T>150{ }^{\circ} \mathrm{C}$ is likely to originate from the degradation of the morphology resulting from the formation of large PCBM aggregates. ${ }^{25}$

In conclusion, we have demonstrated ambipolar FETs using a 1:1 mixture of rr-P3HT and PCBM and using Al for the source and drain electrodes. For devices annealed at $150{ }^{\circ} \mathrm{C}$ for $10 \mathrm{~min}$, the hole mobility is $\mu_{h}=1.7$ $\times 10^{-3} \mathrm{~cm}^{2} / \mathrm{V} \mathrm{s}$ and the electron mobility is $\mu_{e}=2.0$ $\times 10^{-3} \mathrm{~cm}^{2} / \mathrm{V}$ s. The improved ambipolar performance is attributed to the increased crystallinity of the rr-P3HT and PCBM and to the improved contact between the active layer and the metal electrode ( $\mathrm{Al})$, both induced by the thermal annealing process.

This work was supported by the Samsung Advanced Institute of Technology.

${ }^{1}$ G. Horowitz, Adv. Mater. (Weinheim, Ger.) 10, 365 (1998).

${ }^{2}$ B. Crone, A. Dodabalapur, Y.-Y. Lin, R. W. Filas, Z. Bao, A. LaDuca, R. Sarpeshkar, H. E. Katz, and W. Li, Nature (London) 403, 521 (2000).

${ }^{3}$ H. E. A. Huitema, G. H. Gelinck, J. B. P. H. van der Putten, K. E. Kuijk, C. M. Hart, E. Cantatore, P. T. Herwig, A. J. J. M. van Breemen, and D. M. de Leeuw, Nature (London) 414, 599 (2001).

${ }^{4}$ C. D. Sheraw, L. Zhou, J. R. Huang, D. J. Gundlach, T. N. Jackson, M. G. Kane, I. G. Hill, M. S. Hammond, J. Campi, B. K. Greening, J. Francl, and J. West, Appl. Phys. Lett. 80, 1088 (2002).

${ }^{5}$ T. D. Anthopoulos, D. M. De Leeuw, E. Cantatore, S. Setayesh, E. J. Meijer, C. Tanase, J. C. Hummelen, and P. W. M. Blom, Appl. Phys. Lett. 85, 4205 (2004).

${ }^{6}$ Th. B. Singh, S. Günes, N. Marjanović, N. S. Sariciftci, and R. Menon, J. Appl. Phys. 97, 114508 (2005).

${ }^{7}$ E. J. Meijer, D. M. De Leeuw, S. Setayesh, E. V. Veenendaal, B. -H. Huisman, P. W. M. Blom, J. C. Hummelen, U. Scherf, and T. M. Klapwijk, Nat. Mater. 2, 678 (2003).

${ }^{8}$ H. Sirringhaus, N. Tessler, and R. H. Friend, Science 280, 1741 (1998).

${ }^{9}$ H. E. Katz and Z. Bao, J. Phys. Chem. B 104, 671 (2000).

${ }^{10}$ C. D. Dimitrakopoulos and P. R. L. Malenfant, Adv. Mater. (Weinheim, Ger.) 14, 99 (2002).

${ }^{11}$ A. Babel and S. A. Jenekhe, Adv. Mater. (Weinheim, Ger.) 14, 371 (2002).

${ }^{12}$ R. C. Haddon, A. S. Perel, R. C. Morris, T. T. M. Palstra, A. F. Hebard, and R. M. Fleming, Appl. Phys. Lett. 67, 121 (1995).

${ }^{13}$ C. Rost, D. J. Gundlach, S. Karg, and W. Rieß, J. Appl. Phys. 95, 5782 (2004).

${ }^{14}$ Y. Hayashi, H. Kanamori, I. Yamada, A. Takasu, S. Takagi, and K. Kaneko, Appl. Phys. Lett. 86, 052104 (2005).

${ }^{15}$ K. Tada, H. Harada, and K. Yoshino, Jpn. J. Appl. Phys., Part 2 36, L718 (1997).

${ }^{16}$ A. Babel, J. D. Wind, and S. A. Jenekhe, Adv. Funct. Mater. 14, 891 (2004).

${ }^{17}$ A. Dodabalapur, H. E. Katz, L. Torsi, and R. C. Haddon, Appl. Phys. Lett. 68, 1108 (1996).

${ }^{18}$ E. Kuwahara, Y. Kubozono, T. Hosokawa, T. Nagano, K. Masunari, and A. Fujiwara, Appl. Phys. Lett. 85, 4765 (2004).

${ }^{19}$ M. L. Chabinyc, J.-P. Lu, R. A. Street, Y. Wu, P. Liu, and B. S. Ong, J. Appl. Phys. 96, 2063 (2004).

${ }^{20}$ W. Ma, C. Y. Yang, X. Gong, K. Lee, and A. J. Heeger, Adv. Funct. Mater. 15, 1617 (2005).

${ }^{21}$ S. Cho, M. Surin, J. Yuen, G. Wang, R. Lazzaroni, D. Moses, K. Lee, and A. J. Heeger (unpublished).

${ }^{22}$ X. Yang, J. Loos, S. C. Veenstra, W. J. H. Verhees, M. M. Wienk, J. M. Kroon, M. A. J. Michels, and R. A. J. Janssen, Nano Lett. 5, 579 (2005).

${ }^{23}$ T. J. Savenije, J. E. Kroeze, X. Yang, and J. Loos, Adv. Funct. Mater. 15, 1260 (2005).

${ }^{24}$ M. Reyes-Reyes, K. Kim, and D. L. Carroll, Appl. Phys. Lett. 87, 083506 (2005).

${ }^{25}$ H. Hoppe, M. Niggemann, C. Winder, J. Kraut, R. Hiesgen, A. Hinsch, D. Meissner, and N. S. Sariciftci, Adv. Funct. Mater. 14, 1005 (2004). 